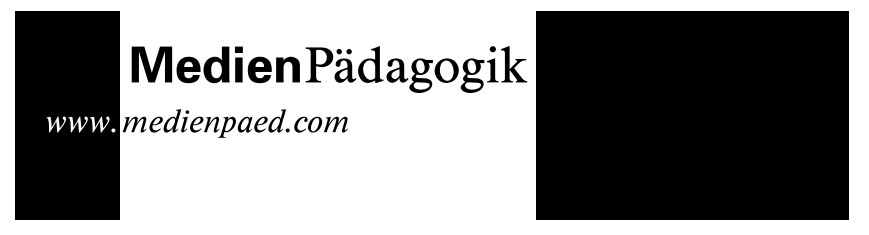

Claudia de Witt

17.3.2000

\section{Medienbildung für die Netz-Generation}

Ich war gerade mal achtzehn - habe ich an meiner ersten Konferenz zum Thema Computersicherheit teilgenommen und einen technischen Fehler in einer Präsentation bemerkt.

Der Typ am Rednerpult hatte viele, viele Jahre lang bei der National Security Agency und beim Militär an Sicherheitsprogrammen gearbeitet, und da stehe ich mit achtzehn - achtzehn - vor all diesen Leuten auf und weise ihm einen technischen Fehler nach.

aus: Hard, Soft \& Wet $(1998,267)$

\section{Einleitung}

Computerspezialisten sind schon lange nicht mehr nur ältere ausgebildete Fachleute mit langjähriger Erfahrung, sondern auch Jugendliche, die den Computer beherrschen. Sie benutzen ihre Computerkenntnisse, um Spass zu haben, sich auszuprobieren, andere zu beeindrucken oder um Kontrolle auszuüben. «Oft sind es die jüngeren, die sich den neuen Wahrnehmungsweisen mit Neugier und nicht mit Abwehr stellen; sie sind es auch, die zumindest in den Bereichen Medien, Konsum, Freizeit, Mode überdurchschnittlich gut Bescheid wissen und auch für die ältere Generation Orientierungssignale setzten ... Vieles lernen die Älteren heute von den Jüngeren. Von den Freizeitstilen bis zu den Medien: Es sind die jungen Menschen, die Bescheid wissen» (Baacke 1997, 23). Diesem neuen Beziehungsverhältnis geht auch der amerikanische Unternehmensberater Tapscott nach, der eine Reihe von Kompetenzen bereits der jüngeren Generation, den NetKids, zuspricht, die sich gegenüber der Fernseh-Generation durch ihr Aufwachsen mit einem sich geradeerst entwickelnden interaktiven Kommuni- kationsmedium auszeichnet. Sie ist die erste Generation, die inmitten digitaler Medien aufwächst. Diese Generation der 4- bis 29-jährigen sei besonders neugierig, innovativ, teambezogen und leistungsorientiert.

Diese Kompetenzen in diesem Alter machen Eindruck. Und es stellt sich die Frage, ob die pädagogische Vermittlung von Medienkompetenz oder Medienbildung überhaupt noch notwendig ist. Um der Frage auf den Grund zu gehen, wird daher die Interaktionskultur der Netz-Generation näher betrachtet, in deren Mittelpunkt die Wechselwirkung zwischen den Handlungen der Heranwachsenden und den Strukturen der neuen Medien steht. Es wird anschliessend herausgearbeitet, dass die Netz-Generation zwar selbstbewusster als die Fernseh-Generation ist, aber Ansätze zu einem neuen Erziehungs- und Lernverhältnis (vgl. Baacke 1997) und insbesondere Überlegungen zur Medienbildung in einer neuen interaktiven Kultur des Lernens gerade angebracht sind.

\section{Die Interaktionskultur der Netz-Generation}

Die Netz-Generation wächst heute selbstverständlich mit digitalen Medien auf und wird daher auch als Generation N oder Generation@bezeichnet. Sie unterscheidet sich in ihren Fähigkeiten von denen der Baby-Boomer («alle die in den Jahren 1946 bis 1964 Geborenen», Tapscott 1998, 38) und fühlt sich nicht von der gesellschaftlichen Entwicklung ausgeschlossen, sie schafft sich vielmehr eine eigene Lebenskultur und grenzt dabei eher ihre Elterngeneration aus. Die heutigen Jugendlichen sind «hellwach, problembewusst, konzentriert und haben ganz offensichtlich die Dinge im Griff» (Tapscott 1998, 128). Sie zeichnen sich durch eine Akzeptanz der Verschiedenheit, durch Neugierde und durch eine Bestimmtheit bzw. ein Selbstvertrauen aus, das durch das Ausprobieren verschiedener Rollen im Netz gestärkt wird (vgl. <www.growingup digital.com/ngthink.html〉).

Das Internet ist für sie genauso ein selbstverständlicher Bestandteil ihres Lebens wie für die Babyboomer Fernsehen und Telefon. Die Aktivitäten der Netz-Generation im Netz umfasst eine breite Palette. Favorisiert wird das Chatten - mit kinderspezifischen Internet-Filtern (z. B. Freezone) - und das Versenden elektronischer «Postkarten». Die Heranwachsenden partizipieren zudem aktiv am Internet durch ihre eigenen Homepages. Für die Jüngeren stehen vorgefertigte Formate zur Verfügung, wie z. B. bei 〈KidsCom〉, 〈cyberisle.org〉 oder 〈cityspace.org〉, die älteren entwickeln eigene Designs und nutzen dabei die vielfältigen Möglichkeiten von HypermediaSystemen und die Kommunikationsmöglichkeiten wie Email, Chat oder 
Newsgroup. Es ist auch zu vermuten, dass sich der E-Commerce bei den Jugendlichen zunehmend vergrössern wird.

Die Interaktionskultur der Netz-Generation lebt von Vernetzungen, Transformationen und Molekularisierungen und damit von einer grösseren Beweglichkeit. Dabei ist zu beachten, dass die Fähigkeiten der jeweiligen Generationen in Beziehung zu den Strukturen der vorrangig genutzten Medien stehen und nicht zuletzt durch diese erst hervorgerufen werden. «Das Medium bestimmt die Kultur ... Im Zentrum der Netz-Generation steht die Interaktivität» (Tapscott 1998, 117).

Es gibt plausible Annahmen, die einen kausalen Zusammenhang zwischen den Strukturen von Medien und die Ausbildung von Kompetenzen herausstellen. Bereits 1987 hat Greenfield die These aufgestellt, dass nicht nur das Fernsehen sondern auch der Computer für Kinder und Jugendliche förderlich ist. Sie geht davon aus, dass erlernte Fähigkeiten mit einem elektronischem Medium dem Heranwachsenden zu weiteren Fähigkeiten verhelfen, mit später eingesetzten elektronischen Medien umzugehen (vgl. Greenfield 1987). So baue der Computer auf den dynamischen Qualitäten des Fernsehens auf, ergänze die Qualität der Bewegung aber um Interaktivität und Programmierbarkeit. Durch die Aufnahme von Informationen mit Hilfe verschiedener Medien lerne man, ein bestimmtes Thema aus unterschiedlichen Perspektiven wahrzunehmen. Auch Schnoor (1992,193) stellt heraus, dass «technische Bilder ... das Aufwachsen von Kindern und Jugendlichen in umfassender Weise verändert (haben). Sie beeinflussen, indem sie den Blick auf die Welt verändern, ihr Denken, Fühlen und Handeln. Technische Bilder beanspruchen für sich, universell zu sein und alle Sinne anzusprechen. Doch sie verbergen, dass sie nur einen informierten Wahrnehmungsmodus zulassen».

Die Interaktivität der neuen Medien mit ihren nicht-linearen HypertextStrukturen macht die Heranwachsenden nicht nur zu Empfängern, sondern immer auch zu aktiven Sendern von Informationen. «Das Netz scheint ihnen ... ein geeignetes Werkzeug zu bieten, mit dem sie sich als unabhängige, eigenständige Individuen Gehör verschaffen können. Diese Art der Interaktion fördert Kinder in ihrem Wachstum und verlangt von ihnen, eigene Werte zu entwickeln, ihr Urteilsvermögen zu schulen, zu analysieren, $\mathrm{zu}$ evaluieren und zu kritisieren oder anderen irgendwie behilflich zu sein. Die Interaktion fördert auch das Selbstbewusstsein, obwohl die Kids auch ihre Cyberfreunde im Netz häufig um emotionale Unterstützung bitten. Vor allem Mädchen scheinen die Unterstützung Gleichaltriger im Cyberspace sehr zu geniessen und auch zu brauchen» (Tapscott 1998, 88). Die NetzGeneration wird auch eher ihre eigenen Unternehmen gründen und führen als alte träge Unternehmen umzugestalten.

Für Tapscottt beruht das neue Selbstbewusstsein der Netz-Generation auf den interaktiven Strukturen der digitalen Welt. Während die Baby-Boomer vom Fernsehen beeinflusst wurden, lernt die Netz-Generation auf interaktive Weise. Und zum ersten mal seien Kinder und Jugendliche auf einem ganz zentralen Feld gebildeter als ihre Eltern. Durch die Interaktion mit dem Medium sind Aktivitäten erfordert, Lesen, Schreiben und die damit verbundenen kognitiven Leistungen würden spielerisch eingeübt. Vermutlich entwickelt sich dabei eine neue Jugendsprache mit einer eigenen Form der Verbalität (vgl. 〈http://www.planetpals.com/smiley.html ).

Durch ihre aktive Kontrolle ihrer Medien entwickelten sich die Heranwachsenden auch viel schneller. Dies bedeute aber keineswegs das Ende der Kindheit. «Stattdessen hat sich den Kindern eine neue Spielwelt eröffnet», sie definieren sich über ihre Beziehung zu ihren vorrangigen Medien und haben dabei «völlig neuartige und wirksame Methoden des Fragens, Forschens, der Einflussnahme und des Spielens zur Verfügung» (Tapscott 1998, 18) (vgl. z. B. 〈www.kindernetz.de〉).

Die folgenden 10 Fähigkeiten sind nach Tapscott besonders charakteristisch:

1. Unabhängigkeit und Autonomie

2. Emotionale und intellektuelle Offenheit

3. Einbeziehung des sozialen Lebens

4. Freie Meinungsäusserung und keine Tabus

5. Innovation

6. Sorge um die eigene Reife

7. Forschergeist

8. Unmittelbarkeit und Echtheit

9. Abneigung gegen Unternehmensinteressen

10. Echtheit und Vertrauen ${ }^{1}$

Entgegen vieler Meinungen sei diese Generation nicht selbstsüchtig, zynisch und materialistisch, obwohl sie materiellen Luxus schätze. Sie verfüge über mehr Wissen als jede frühere Generation und soziale Probleme seien ihr nicht gleichgültig. Der Generation N komme eben zugute, dass sie eine Generation der Lernenden ist.

1 Wie diese Fähigkeiten der Netz-Generation Unternehmen verändern, stellt Tapscott unter 〈http://www.flexible-unternehmen.com/fb9900121.htm〉 dar. 
Allerdings würde die digitale Kluft zwischen Informierten und Nicht-Informierten immer weiter klaffen und keinesfalls verschwinden (vgl. Tapscott 1998, 337). Wichtig sei es einen Netz-Zugang nicht nur zu besitzen, sondern auch etwas daraus zu machen. Mit den besseren Kenntnissen ist auch eine höhere Motivation verbunden. Es müsse auch mit den Vorurteilen aufgeräumt werden, dass die häufige Beschäftigung mit dem Internet zu sozialer Isolation führe oder die Netz-Generation an mangelnder Konzentrationsfähigkeit leide, denn erstens werde das «Netz ... als kommunikatives Medium empfunden» und zweitens handle es sich um eine andere Form der Konzentrationsfähigkeit, die sich auf die Fähigkeit zum MultiTasking bezieht.

Während Tapscott davon spricht, dass die Netz-Generation ein «völlig neuartiges Gemeinschaftsmodell entwickelt und bereits in jungen Jahren ernst zunehmende Impulse in Richtung eines sozialen und wirtschaftlichen Wandels geben (vgl. Tapscott 1998), ist der Freizeitforscher Opaschowski verhaltener. ${ }^{2}$ Noch gäbe es mehr Leser als Computerfreaks und die Internet-Revolution habe in Deutschland noch nicht stattgefunden. Und bei aller Euphorie hat der Einfluss der digitalen Medien auch problematische psychische und soziale Folgen für den Heranwachsenden.

\section{Problematische Auswirkungen der Mediatisierung}

\section{auf das Wesen und das Sozialverhalten der Netz-Generation}

Pädagogen sollten nicht darauf vertrauen, «dass die Medien bei den Kindern «problemlos Verwendung finden〉, weil sie vermeintlich selbständiger und kompetenter mit den Medien umgehen können, als die Erwachsenen ihnen zutrauen» (Opaschowski 1999, 88). Sie sind Reizüberflutungen nicht nur durch Computererlebnisse - ausgesetzt. Als «Konfetti»-Generation nähmen sie die vielfältigen Impressionen bruchstückhaft und oberflächlich auf. Psychosoziale Folgen sind zu erwarten. «Das Kind wird zum Scanner, d. h. das Aufwachsen in einer reizüberfluteten Umwelt zwingt das Kind, auch das eigene Leben zu scannen» (Opaschowski 1999, 77). Entgegen der Auffassung von Tapscott sind die Heranwachsenden nach Opaschowski nicht nur einem Erreichbarkeitswahn erlegen, sondern auch einem Informationsstress ausgesetzt, der zulasten von Konzentration und

2 Dabei ist zu berücksichtigen, dass sich Tapscotts Untersuchungsergebnisse auf Amerika beziehen, wo das Internet bereits intensiver genutzt wird als in Deutschland und dort bereits grösseren Einfluss auf Wirtschaft und Gesellschaft hat Deutschland befindet sich eher in einem Anfangsstadium (vgl. Opaschowski 1999).

$5 / 12$
Aufmerksamkeit geht. Einen Ausweg aus dieser Beschleunigung sieht Opaschowski in einer «Info-Ökonomie»: «Die Problemlösung für die Zukunft kann nur eine sich selbst schützende Info-Ökonomie sein, die zeitweilig zur freiwilligen Einfachheit zurückkehrt ... Das bedeutet: Bewusst zu langsameren Möglichkeiten der Kommunikation zurückkehren und nicht mehr unbedingt alle Info-Angebote nutzen. Wer sich vom Erreichbarkeitswahn befreien will, muss reduzieren, begrenzen und gelegentlich auch rigoros abschalten können» (Opaschowski 1999, 131).

Die Generation @ ist nicht nur eine Generation «flexibler Drifter», sondern auch «genervte Einzelgänger», die den Computer als Rückzugsnische benützten: «Die Generation @ driftet durch das Dasein, lebt temporär und kommt kaum zur Ruhe. ... Sie verliert das Gefühl für die Balance zwischen schnell und langsam, Anspannung und Entspannung. Sie verlernt, in mittlerem Tempo zu leben ... In der Mitte, also bei einem Mittelmass an Zeitdruck, ist das Wohlbefinden am grössten. Die genervte Generation @, die viel zuviel gleichzeitig macht und sich übermässig oft im Stress befindet, wird zunehmend mit gesundheitlichen Problemen zu kämpfen haben» (Opaschowski 1999, 145). Der kompetente User ist ein Individualist par excellence.

Es entwickelt sich eine «Kurzzeit-Konzentrations-Kultur»: «Immer mehr Menschen sind dann nicht mehr in der Lage, sich über längere Zeiträume mit den gleichen Dingen zu beschäftigen. So wächst folgerichtig eine Generation heran, die von klein auf ein «K.K.K.»-spezifisches Konsumentenverhalten erlernt («ständig wechselnde Bekanntschaften, viele und wechselnde Kontakte würden zur Manie, Kontakten wird zum Zugzwang... Oberflächlichkeit der Beziehungen ist vorprogrammiert»(Opaschoski 1999, 79). Psychosoziale Folgen sind Entwicklungsdefizite und Sprachauffälligkeiten, Beeinträchtigung der kommunikativen Kompetenz (reduzierter Wortschatz, Probleme bei der Anwendung grammatischer Regeln, Schwierigkeiten beim Verstehen und Formulieren von Sätzen, Nervosität und Aggressivität, Hyeraktivität und Konzentrationsmangel. Daher gehört für Opaschowski eine neue Lehrerkompetenz: «Motivations- und Animationsfähigkeiten sowie die Kompetenz zu Methodenwechsel und variantenreichen Unterrichtsformen werden dann zum pädagogischen Bestandteil jedes Lehrers bzw. zum Grundbestandteil jeder Lehreraus- und -fortbildung gehören müssen» $(1999,85)$. 


\section{Die Netz-Generation - eine postmoderne Generation} der Schein-Selbständigen?

Durch ihren selbstverständlichen Umgang mit den neuen Medien entwickelt sich bei der Netz-Generation eine Selbständigkeit und darüber ein neues Selbstbewusstsein. Aber es muss doch der Frage nachgegangen werden, ob diese von Tapscott beschriebene Selbständigkeit der heranwachsenden Generation nicht nur eine «Schein-Selbständigkeit» ist. Dies geschieht in Anlehnung an Jostocks Methode, die über einen Vergleich von aufklärerischer und postmoderner Bildung den Entwurf einer spätmodernen Bildungskindheit skizziert.

a) Als erstes postmodernes Element tritt das neue Generationenverhältnis in Erscheinung, das sich in einer «Entdifferenzierung der Generationen» bzw. in einer «Äquilibration» äussert: So steht die Bezeichnung «Generation-Gap» für die Kluft zwischen einer den neuen Medien aufgeschlossenen Jugend und einer tendenziell verunsicherten älteren Generation. Die jugendliche Generation verfügt in diesem zukunftsträchtigen Bereich bereits oft über mehr Kompetenzen als Erwachsene (vgl. Schwab/Stegmann 1999, 19). Unbestritten ist ein offeneres Verhältnis, bei dem die ältere Generation ihr umfangreicheres Wissen und moralische Reife einbringen kann (vgl. Aufenanger 2000).

Es ist aber in Frage zu stellen, ob die Heranwachsenden nicht «nur scheinbar zu gleichberechtigten und mündigen Partnern geworden (sind)» (Jostock 1999, 92). Denn es wird nämlich nicht genug berücksichtigt, dass Heranwachsende sich nach einer Entwicklungslogik entfalten und in jeder Altersstufe Grenzen des vernünftigen Verstehens bestehen. Die Netz-Generation experimentiert zwar mit den Möglichkeiten des Internet und WorldWideWeb, aber es mangelt an der Fähigkeit, gesellschaftliche Gefährdungen vernünftig zu durchschauen und vernünftig entgegentreten zu können (vgl. Jostock 1999).

b) Die Selbständigkeit der Netzgeneration steigt immer früher, weil ihr Freiheitsspielraum durch die Souveränität mit den neuen Medien erweitert wird und sie durch ihren Vorsprung an Netz-Kompetenz an scheinbarer Freiheit gewinnt. Denn Individualisierungstendenzen und gesteigerte Selbständigkeit verlangen selbständige Entscheidungen beispielsweise auch bei der Auswahl sozialer Beziehungen im Netz. Aber es kann sich dabei um eine «früher einsetzende äusserliche Verselbständigung» handeln, die noch keine tatsächliche Selbständigkeit bedeutet. Eine individuell-vernünftige Selbständigkeit wäre erst dann erreicht, wenn sie mit einer verstärkten vernünftigen Selbstbestimmung und Selbsttätigkeit im Zusammenhang stehen würde (Jostock 1999, 97).

c) Da Letztbegründungen heute überholt sind, kommt es auf gut begründbares Urteilen und Handeln an. Die Dominanz des Verhandelns und Aushandelns ist eine weitere postmoderne Bedingung der Netz-Generation: Individuelles Handeln wird nur scheinbar dadurch gefördert, dass von Heranwachsenden eine Handlungssouveränität durch Verhandlungsund Aushandlungsprozesse erworben wird. Sie würden vielleicht argumentieren lernen, «ohne jedoch schon die tatsächlichen Gründe für die eine oder andere Wahlentscheidung in ihrer gesamten Tragweite einsehen zu können» (Jostock 1999, 99). Sie lernen vielmehr beliebig zu entscheiden.

d) Unter den Individualisierungsbedingungen der Planbarkeit und Konstruierbarkeit werde Selbstliebe zur Eigenliebe, die dazu führen kann, dass die Freiheit des anderen keine Rolle mehr spielt. Dies weist nur auf eine scheinbare Vernünftigkeit hin.

e) Postmodernes Merkmal ist auch die Zunahme an Reflexivität. Die Windows-Generation braucht eine hohes Mass an Reflexivität, «um soziale Erfahrungen und Bedeutungen im stetigen Wandel bearbeiten zu können. Komplexe und soziale Strukturen der sozialen Welten machen dies notwendig. Es müssen permanent Entscheidungen getroffen werden, die an sich krisenhaft sind. Der einzelne wird somit sensibler für die Reflexion seiner selbst» (Schwab/Stegmann 1999, 29). Aber es werden unter Individualisierungsbedingungen Entscheidungen von Heranwachsenden verlangt, die sie noch nicht bewältigen können. Sie handeln und entscheiden dann nicht vernünftig, sondern anhand von «Moden, Meinungen, Konsumverlockungen» als Entscheidungsersatz (Jostock 1999, 100). Hinzu kommt die Zunahme an verdeckter Herrschaft, wenn die ältere Generation Entscheidungs- und Handlungsleistungen an die Heranwachsenden deligiert (vgl. Jostock 1999).

\section{Auf dem Wege zu einer pragmatischen Medienbildung}

Internet, WorldWideWeb und Multimedia ermöglichen die globale asynchrone Kommunikation und Kooperation. Man kann mit ihnen jederzeit überall sein. Die Netz-Generation wird zum Leitbild einer vom Computer geprägten Gesellschaft, das flexible und mobile, interdisziplinär und global handelnde, leistungsfähige, effektive und erfolgreiche Menschen repräsentiert. Die optimistische Position geht davon aus, dass die digitalen Medien 
selbstbestimmtes und eigenverantwortliches Handeln hervorrufen. Es bestehen vielfältige Möglichkeiten Wissen anzuwenden und auszuprobieren. Aber dies ist auch gleichzeitig mit Problemen verbunden. Z. B. setzen die vielfältigen Möglichkeiten der neuen Medien mehr als zuvor die Fähigkeit voraus, Informationen kritisch zu hinterfragen. Durch die Hypertextstrukturen der Neuen Medien können gerade - im Gegensatz zu Tapscotts Optimismus - Orientierungslosigkeit, Konzentrationsschwäche oder unsystematische Lernvorgänge zur Regel werden.

Für die Pädagogik sind durch die rasanten Medienentwicklungen Veränderungen hinsichtlich der Aspekte Mobilität, Miniaturisierung und Digitalisierung, Integration und Globalisierung sowie Kommerzialisierung zu erwarten (vgl. Aufenanger 2000,6). Diese Veränderungsprozesse führen wieder zu einer Aufwertung des Bildungsbegriffs, «der nun auf die durch Medien geprägte Wissensgesellschaft hin ausgerichtet sein sollte» (Aufenanger 2000,7). Denn der Begriff der Medienkompetenz sei doch sehr eingeschränkt und besser durch «Medienbildung» zu ersetzen, da damit auch die gesellschaftliche Dimension (z. B. Fragen der sozialen Benachteiligung) und «Fragen nach unserem Selbst und unserer Identität auch im Medienzeitalter unabhängig von Medien» gestellt werden können (Aufenanger 2000,7).

Auch Baacke hält den Begriff der Medienkompetenz trotz seiner Differenzierungen (vgl. Baacke 1999b; 2000) pädagogisch unspezifisch und plädiert dafür, in den Begriff der Medienkompetenz die «Dimensionen von Erziehung und Bildung ... einzudenken. (Denn) wer von Medienkompetenz redet, muss gleichzeitig davon reden, wie diese zu vermitteln sei und wo das Subjekt in seiner sich ausbildenden oder sich ausgebildet habenden Selbstverantwortlichkeit seinen kommunikativen Status bestimme» (Baacke 1999a, 24). Kommunikative Kompetenz bekommt durch den Einfluss der neuen Medien eine neue Bedeutung: Die Medien sind einerseits verantwortlich für die Veränderung von Erziehung und Lernen, andererseits verhelfen sie zu neuen «Beziehungs-Formationen». Über einen gegenseitigen «Austausch von Kompetenzen» entwickelt sich eine «Welt allumfassender Partizipation» (Baacke 1997, 23ff). Aufgrund der Tendenz zur globalen Partizipation entsteht für Baacke eine «neue Beziehungs-Formation» im Sinne eines gegenseitigen «Austauschs von Kompetenzen» (Baacke 1997, 23). Der neue Sinn von Erziehung und Bildung und das Verhältnis von Erziehenden und zu Erziehendem besteht daher in einer «Kreisbewegung offener Kommunikation, in der wir unsere jeweiligen Kompetenzen freimütig und bereitwillig austauschen und insofern tatsächlich Herrschaft abbauen oder doch einschränken. Mit neuen Wahrnehmungsformen umgehen, die neuen Kommunikationstechniken angemessen bedienen können: dies ist eine Lernaufgabe, die Kindern und ihren Lehrern in gleicher Weise gestellt ist» (Baacke 1997, 25).

Was der Netz-Generation fehlt ist die Fähigkeit zum «richtigen» Umgang mit Informationen und bedeutungsvollem Wissen, der nur durch reflektierende Urteilskraft (vgl. Sandbothe 1999) und ihren Gebrauch erfolgen kann. Da Bildung in der Mediengesellschaft nichts Statisches ist, sondern ein offener, dynamischer Prozess und Computerwissen keine Gewissheit und keinen Konsens verspricht (vgl. Glotz 1999), ist die Bildung von Orientierungswissen und kritischer Distanz gegenüber den immer rascheren Modernisierungstendenzen so wichtig wie nie zuvor (vgl. «www.forum-bildung.de $)$.

In Zeiten gesellschaftlicher Veränderungen bekommen gerade zeitunabhängige Kompetenzen Bedeutung. Dazu gehören neben kommunikativen auch methodische Kompetenzen, die die Grundlage für eine von Medien geprägten interaktive Lernkultur bilden. Bildungsziel in einer Gesellschaft, die durch die interaktiven Strukturen der Medien beeinflusst wird, ist die Fähigkeit, durch kommunikative Organisationsprozesse Urteils- und Verständnisvermögen zu entwickeln und dabei Eigenverantwortung zeigen und kommunikative Zielvereinbarungen durchführen zu können.

Eine pragmatische Medienbildung betont den Gebrauch der Medien und verfolgt nicht Methoden, die auf Akzeptanz abzielen, sondern eine Methode der Kontrolle, durch die Handlungsprozesse mit kritischer Urteilsfähigkeit geleitet werden. Eine pragmatische Methode hat nichts Dogmatisches, sondern schliesst vielmehr kreatives Experimentieren, Risikofreude genauso wie kontrollierende Reflexion ein. Dabei kann sich eine eigenständige Beurteilungskompetenz nur dann entwickeln, wenn sie sich an Situationen, genauer an den sowohl gegenständlichen, objektiven als auch zuständlichen, subjektiven Bedingungen jeder Situation orientiert. Pragmatische Medienbildung basiert auf einer Situationsorientierung: «Ob sich jemand als medienmündig erweist, hängt damit weniger von den normativen Zielvorstellungen der Medienmündigkeit oder -kompetenz ab, als von der konkreten Bewältigung von Handlungs- und Lebenssituationen» (Mikos 1999, 22).

Über eine interaktive Auseinandersetzung mit den objektiven und den subjektiven Bedingungen einer Situation organisiert sich Erfahrung. Über 


\section{MedienPädagogik}

diese Organisation von Erfahrung vollzieht der Heranwachsende damit eine Leistung, seine Interessen in Relation zu den Mitteln seines Handelns und den antizipierten Folgen zu bringen. Dies meint auch die Fähigkeit, Kontrolle und Orientierung in den Handlungen einzuüben und damit die Möglichkeit zu haben, seine Interessen und Handlungen zu verändern. Damit die Netz-Generation tatsächliche und dauerhafte Selbständigkeit erwirbt, ist die Bildung urteilsfähiger Erfahrungsprozesse notwendig, die Skepsis, Kritik und intelligente Handlungen bei Problemlösungs- und Organisationsprozessen einschliessen.

\section{Literatur}

Aufenanger, Stefan. Mediale Visionen und die Zukunft der Medienpädagogik. In: Medien praktisch 1/2000, 4-8

Baacke, Dieter. Schulen im Strom gesellschaftlicher Kommunikation. In: Pädagogik 6/97, 23-25

Baacke, Dieter. «Zum Konzept und zur Operationalisierung von Medienkompetenz» Unter 〈http://www.gmk.medienpaed.de/aufs/auf002.htm〉 Stand: 16.02 .00 (vgl. auch Baacke, Dieter. «Medienkompetenz»: theoretisch erschliessend und praktisch folgenreich. In: medien+ erziehung 1/1999b, 7-12)

Baacke, Dieter. Im Datennetz. Medienkompetenz (nicht nur) für Kinder und Jugendliche als pädagogische Herausforderung. In: Baacke, D./Lauffer, J./Thomsen, M. (Hrsg.). Ins Netz gegangen. Internet und Multimedia in der ausserschulischen Pädagogik. Bielefeld 1999a, 14-27

Glotz, Peter. Die beschleunigte Gesellschaft. Kulturkämpfe im digitalen Kapitalismus. Kindler München 1999

Greenfield, Patricia. Kinder und neue Medien. Psychologie-Verl.-Union München 1987 Jostock, Simone. Kindheit in der Moderne und Postmoderne. Eine bildungstheoretische und sozialwissenschaftliche Untersuchung. Leske+Budrich Opladen 1999

McGrath, Melanie. Hard, Soft \& Wet. Rowohlt Hamburg 1998

Mikos, Lothar. Ein kompetenter Umgang mit Medien erfordert mehr als Medienkompetenz. In: medien+erziehung 1/1999, 19-23

Opaschowski, Horst W. Generation @ - Die Medienrevolution entlässt ihre Kinder: Leben im Informationszeitalter. Hamburg 1999

Sandbothe, Mike «Globalität als Lebensform. Überlegungen zur Ausbildung einer Internetspezifischen Urteilskraft» 〈http://www.uni-jena.de/ms/global.html〉 Stand: 13.12.99

Schnoor, Detlev. Sehen lernen in der Fernsehgeneration. Leske+Budrich Opladen 1992 Schwab, Jürgen/Stegmann, Michael. Die Windows-Generation. München 1999

Tapscott, Don. Net Kids. Die digitale Generation erobert Wirtschaft und Gesellschaft. Gabler Verlag Wiesbaden 1998 